\title{
Control of Spread of Microorganisms in the Hospital - Back to the Basics of Hand Washing and Glove Use
}

In this issue of the BJID, Korn, et al have identified again the high risk to patients of acquiring a methacillinresistant Staphylococcus aureus infection after entering an Intensive Care Unit (ICU) [1]. 52\% of patients entering the ICU became colonised with the organism, $22 \%$ had documented infections, and over half of those infected died of their infection. The authors concluded that there were no special risk factors for this situation, and that a re-emphasis on basic antisepsis - including hand washing, environmental surface cleaning and barrier protection (gloves) was needed. That conclusion is certainly a correct one and very important. The question remaining, is it really possible to alter infection rates by improving antiseptic procedures? The answer is yes, but how is complex [2].

It is clear we need to do more than following old guidelines or even to creating new ones. We need to re-establish an entirely new culture among health care workers based on careful scientific evidence of the issues, full awareness of the devises available, and practical advice for each hospital setting. Rapid decision making, speed of action, and the most up-to-date medical technological support systems have been the hallmark of our ICUs. In the process some of the most important, and long known steps in preventing the spread of microorganisms have been forgotten, or, at least, placed in a secondary position.

One of the best examples of this situation is the present on-going discussion of the procedure of hand washing by professional staff in hospitals in the USA [25]. Hand washing is but one part of what is termed "hand hygiene". It includes 1) hand washing with nonantimicrobial soap or detergent - a procedure that removes dirt, skin surface and some microbes, 2) soap that contains antiseptic materials, such as chlorohexidine or povidine - a procedure that removes both dirt and microbes, but that is suboptimal with short-termintermittent use, and 3) alcohol-based products - a procedure that effectively kills bacteria and viruses (termed degerming), but does it does not remove dirt [2].
In Brazil, most hospitals use the second approach to hand hygiene, hand washing with antiseptic detergents, in their procedures for hand washing. Since this is an effective approach, the main issue becomes, "How much time is needed to achieve the ideal in antimicrobial killing, and is this time available to a busy health care worker? Is it possible for health care workers to adhere to present detergent soap hand washing recommendation?"

Apparently in the USA, hand washing with medicated soap and water is the method of choice for control of bacterial spread when the staff moves from patient to patient in the hospital environment. Although the present guidelines are under review, the emphasis by the Centers for Disease Control and Prevention (CDC) remains on the use of medicated soap [6]. The authors of two excellent, recent reviews [2,4] called for serious consideration to substitute alcohol hand rub for the hand washing procedure. Some of the arguments raised by Widmer [4] are the following. It is clear that removal of microbes by hand washing requires 1-2 minutes per wash, plus a minute to get to the sink and back. It has also been recorded that nurses have 20 opportunities per hour in which hand washing should be considered [7], therefore, 2-3 minutes x 20 $=40$ 60 minutes per hour is needed for proper hand hygiene - the nurse has somewhere between 0 and 20 minutes to do her job! It is no wonder that several studies show that there is lower than $40 \%$ compliance with hand washing in ICUs [4]. It is no wonder compliance is low - it is impossible!

Several of the reviews of this subject remind us of the sentinel study of this issue by Ignaz Semmelweis in $1847[3,4]$. He showed that hand washing with nonmedicated soap reduced mortality from peripartum fever from $18 \%$ to $3 \%$, but by introducing the use of chlorinated lime solution the epidemic disappeared completely [8]. Over the 150 years since his studies, this last part seems to have disappeared, until the past decade in Europe where a return to alcohol based 
solution for microbial control has been re-established [4]. It is interesting, in the time of incredible advances in microbiology, impressive developments in antimicrobial drugs, and important advances in hospital infection control, that this rather simple requirement to reduce spread of organisms has been overlooked. The only reasonable explanation must be that the culture of our health care community has not been ready to pour alcohol over our hands 20 times per hour. This may have been due to a thought that alcohol causes irritation, allergy, nail discoloration, a special odour, or excessive drying. Skin allergy to alcohol does not occur and after proper instruction regarding how to use an alcohol rub in Europe high levels of compliance and few complaints have arisen. One wonders whether the attitude against use of alcohol is a leftover from the period of alcohol prohibition in the United States in the 1920-30s. Whatever the cause this culture needs to change.

The steps in hand washing are as follows [9]: 1) Go to the sink, 2) let the water run a few seconds,3) wet hands and wrists with the water, 4) take a dose of soap using the elbow or forearm, 5) rub hands and wrists for 10-15 seconds, 6) rinse, 7) dry gently with a paper towel, without rubbing, 8) use paper towel to turn off the faucet, 9) discard the towel without touching the wastebasket. This is the process that takes one minute for step 1, and 1-2 minutes for steps 2-9.

What are the steps with an alcohol rub in a dispenser next to the patient's bed? 1) remove $3 \mathrm{ml}$ of alcohol from the dispenser, 2) rub into hands, 3) let dry. This procedure requires 18-27 seconds [4].

There are other issues to be considered in deciding between hand washing and alcohol rub. One is that alcohol kills the bacteria, but the dead organisms, plus any dirt, remain on the skin. Therefore, when one needs removal of material from the hands, such as after blood or stool contamination, hand washing will still be required. Second, the best concentration of alcohol for bacterial killing is $70 \%$, the best against viruses is over $95 \%$, so careful consideration of the product specifications for use is needed. Third, the concentration of alcohol in any solution can change over time by evaporation thus, careful consideration of the type of dispenser is required. Fourth, absolute alcohol is flammable, and concentrations of alcohol have varied flash points. It has been stated that in Switzerland, where alcohol rub is now commonly used, that no report of a flammable episode has been made during 5 years of observation [4]. These items must be considered in deciding the best dispenser, the alcohol concentration, whether emollients should be added, and ideal situation for its use.

In addition, it is necessary to examine how to combine the alcohol rub with the equally important process of barrier protection by use of and frequent changes of gloves, and the use of antiseptics on environmental surfaces. It has been shown previously that hands are contaminated with the same organisms that are cultured from gloves, and that hand hygiene must be used in addition to frequent changes of sterile gloves $[5,6]$.

Our conclusion, based on the high risk to patients of iatrogenic disease caused by spread of microbes in the hospital, based on our respect for health care workers who could not possibly do their job and follow the present recommendations, and based on the emerging data supporting the use of an alcohol rub, is that it is time for each hospital to review its policies and install bedside, $3 \mathrm{ml}$ alcohol dispensing units. Members of the Infectious Diseases Society should be leaders in this movement to re-examine all aspects of the important study done 150 years ago.

\author{
Roberto Badaró \\ Editor-in-Chief BJID \\ Thomas $C$. Jones \\ Editor BJID
}

\section{References}

1. Korn G.P., Martino M.D.V., Mimica I.M., et al. High frequency of colonization and abscence of identifiable risk factors for methicillin-resistant Staphylococcus aureus (MRSA) in intensive care units in Brazil. Braz Jou Inf Dis 2001;5(1):1-7

2. Larsen, E. Skin hygiene and infection prevention: More of the same or different approaches? Clin Infect Dis 1999;29:1287-94. 
3. Harbarth S. Handwashing - The Semmelweis lesson misunderstood? Clin Infect Dis 2000;30:990-1.

4. Widmer A.F. Replace hand washing with use of waterless alcohol hand rub? Clin Inf Dis 2000;31:136-43.

5. Struelens M.J. Hospital infection control, in Infectious Diseases, Ed. Armstrong and Cohen, Mosby \& Co., London, Chapter 10, Section 3, 1999.

6. Garner J.S. Hospital infection control practicies advisory committee guidelines for isolation precautions in hospitals. Infect Control Hosp Epidemiol 1996; $17: 53-80$.

7. Pittet D., Mourouga P., Perneger T.V. Compliance with handwashing in a teaching hospital. Ann Intern Med 1999;130:126-30.

8. Semmelweis I.P. Die Aetiologie, der Begriff und Prophylaxis des kindbettfiebers Pest. CA Hartelbens VerlagExpedition, Wien \& Leipzig, 1861.

9. Coignard B. Handwashing quality: impact of a special program. Infect Control Hosp Epidemiology 1998; 19:510-3. 